Instituto Internacional de Investigación y Desarrollo Tecnológico Educativo INDTEC, C.A.

DOI: https://doi.org/10.29394/scientific.issn.2542-2987.2017.2.5.6.105-124

OAI-PMH: http://www.indteca.com/ojs/index.php/Revista Scientific/oai

\title{
Programa para la Integración Didáctica de los Contenidos de Educación Ambiental
}

Autora: Johana Carolina González González Universidad Pedagógica Experimental Libertador, UPEL johanavdsg@hotmail.com

Barinas, Venezuela

\section{Resumen}

La presente investigación tendrá como objetivo establecer un programa para la integración didáctica de los contenidos ambientales desarrollados en el primer grado del Grupo Escolar María La Rivas Salas, ubicado en el Municipio Barinas Estado Barinas, para el año escolar 2014-2015. Este artículo se enmarco en una investigación acción participación (IAP), y es bajo una naturaleza de investigación cualitativa. Se tomaron como actores sociales a tres (03) docentes del primer grado de la institución. Como técnica se utilizó la observación participante y la entrevista en profundidad de tipo no estructurada. Para el análisis de la información se procedió a categorizar, triangular y posteriormente teorizar. Igualmente, la fiabilidad y validez para constatar la información. Como conclusiones se obtuvo el diseño, ejecución y evaluación de un programa desarrollado a través de un plan de acción que presentó estrategias para la integración didáctica de los contenidos de educación ambiental organizadas atendiendo a tres dimensiones que permitan coadyuvar al proceso de enseñanza y aprendizaje en el primer grado del Grupo Escolar María La Rivas Salas, a saber: (a) Sensibilización, (b) Desarrollo del conocimiento y, (c) Participación e integración social.

Palabras clave: programa; integración didáctica; educación ambiental. 


\title{
Program for the Didactic Integration of Contents of Environmental Education
}

\begin{abstract}
The present research will aim to establish a program for the didactic integration of the environmental contents developed in the first grade of the Maria La Rivas Salas School Group, located in the Barinas State of Barinas, for the 2014-2015 school year. This article was framed in an action research participation (IAP), and is under a nature of qualitative research. Three (03) teachers of the first grade of the institution were taken as social actors. As technique, participant observation and in-depth interview of unstructured type were used. For the analysis of the information was proceeded to categorize, triangular and later theorize. Likewise, the reliability and validity to verify the information. As conclusions, the design, execution and evaluation of a program developed through an action plan that presented strategies for the didactic integration of the contents of environmental education organized taking into account three dimensions that allow to contribute to the process of teaching and learning in the First grade of the María La Rivas Salas School Group, namely: (a) Awareness raising, (b) Knowledge development and, (c) Social participation and integration.
\end{abstract}

Keywords: program; didactic integration; environmental education.

Date Received: 05-12-2016

Date Acceptance: 01-02-2017 


\section{Modo de Introducción}

Los programas son un conjunto de herramientas para adquirir habilidades y destrezas en la formación y capacitación de los individuos, donde el mismo es el que promueva cambios que van desde la motivación hasta la inducción de solventar una situación determinada.

Es importante destacar la integración didáctica en la práctica de contenidos ambientales es la acción educativa mediante el cual se adquieren conocimientos y experiencias, con la finalidad de comprender, internalizar y traducir acciones, que incluyan valores y actitudes, tanto individuales como colectivas, tendientes a crear una interacción armónica entre el hombre y el ambiente.

Es por ello que la integración de la didáctica en la enseñanza es producto de la experiencia del docente y de la interrelación de la teoría y la práctica del que hacer educativo, lo que incide en la calidad de la docencia que se imparte en las instituciones de educación. Tal como lo señala Salas (2009), que "la integración didáctica conjuga aspectos teóricos, prácticos y normativos que influyen en los procesos de enseñanza y aprendizaje y en la calidad de la educación" (pág. 12). Es decir, que se considera, por lo tanto, el proceso de enseñanza y aprendizaje mediante la organización de las actividades cognoscitivas, referente a la apropiación y construcción del conocimiento, en un espacio experiencial.

En consecuencia, existe una estrecha relación entre la integración didáctica y el proceso de enseñanza y aprendizaje por cuanto, como indica el autor (ob.cit.), "generar modos de enseñanza que faciliten el aprendizaje en el tiempo acrecienta la cualidad innata del ser humano de aprender a aprender" (pág. 16). Por tanto, el análisis del acto didáctico es complejo, debido a que implica asumir consideraciones relacionadas con el para qué enseñar, qué enseñar, cómo enseñar, con qué enseñar, cómo y cuánto tiempo enseñar y, cómo saber de los logros alcanzados en las áreas del conocimiento; de ahí 
que se puede referir que se establece vinculación del saber, para hacer como práctica en la cotidianidad del ser.

Así mismos la educación ambiental como proceso educativo enfatiza la sensibilización sobre los problemas ecológicos y socio culturales que permitan la promoción de acciones con carácter preventivo y remedial, por lo cual como objetivo esencial en la enseñanza se encuentra el desarrollar no solo un saber ambiental, sino también el reconocimiento de los valores implícitos en esta área, presentes para inculcar en la escuela, en el resto de los factores comunitarios e incluso en el propio hogar, todo ello en función de promover un desarrollo sostenible, para satisfacer las necesidades del presente sin comprometer las capacidades de las futuras generaciones, con la finalidad de preservar el medio ambiente local, regional, y nacional de forma global para mejorar de la calidad de vida de las personas.

Es importante señalar que dentro de este contexto, se hace referencia a la enseñanza de la educación ambiental como proceso de carácter educativo, dirigido a formar valores, actitudes, modos de actuación y conductas a favor del ambiente y para la vida; tal como lo expresa Nieves (2011), "la concepción de la educación ambiental explica la transformación de actitudes, conductas, comportamientos humanos y adquisición de nuevos conocimientos, de allí la necesidad de incorporarla en todas las disciplinas del currículo" (pág. 99). Por consiguiente, cuando se valoran los problemas ambientales como una realidad de la globalización, la didáctica ambiental se transforma en una oportunidad para vincular el proceso docente con la solución de esas situaciones a través de la educación participativa y activa.

Del mismo modo, se hace necesario desarrollar y promover en los estudiantes de todos los niveles y modalidades de enseñanza una nueva manera de visualizar el ambiente. De esta manera, señala Sandoval (2012), que: 
La incorporación de la temática ambiental en los diferentes niveles de la educación, pretende solventar la carencia de una formación ambiental y, la necesidad de relacionar las vivencias del alumno o la alumna con sus experiencias escolares, mediante la introducción en el currículo de una serie de temas que están vivo en la sociedad y que, por su importancia y trascendencia, en el presente y en el futuro, requieren de una respuesta educativa adecuada (pág. 88).

Es decir, la incorporación del conocimiento ambiental en los planes de estudio y el tratamiento de esta, desde los ámbitos conceptual, procedimental y actitudinal constituyen un punto de referencia en el momento de diseñar estrategias y programas de educación ambiental, que le confieran un carácter práctico a la didáctica ambiental presentándola como valiosa, útil y renovadora; que sirva a la educación de los ciudadanos para la comprensión e interpretación de la relación naturaleza hombre sociedad.

Cabe destacar que se requiere de un docente con una alta preparación, capaz de desarrollar en el proceso de formación de sus educandos la capacidad de selección de alternativas de solución de los problemas que se presentan en la escuela, el hogar y la comunidad, para lograr este empeño en el desarrollo de la educación ambiental y con ello incorporar los contenidos ambientales desarrollados en el proceso de enseñanza aprendizaje requieren vincular los elementos del contexto natural con el entorno en el que se desenvuelve el individuo, así como la interrelación e interdependencia de éstos con lo cultural, social, político, económico, educativo, y en los nuevos tiempos espiritual. Es decir, que la finalidad más importante en la enseñanza de la educación ambiental es lograr el desarrollo de las potencialidades y la sensibilización ciudadana.

De esta manera, la educación ambiental entendida con esta visión requiere de modelos metodológicos, no sólo de análisis sino de acción y participación, la metodología ambiental debe ser experimental y dialéctica en 
el análisis de la realidad con la que ha de estar en contacto el sujeto. Por tanto, la búsqueda en la didáctica de nuevos métodos para hacer la educación ambiental más activa, amena y contemporánea se ha convertido en una necesidad de encuentro de técnicas, accionares y, estrategias participativas, entre otros que permitan al docente la generación de ambientes de aprendizajes acordes con los tiempos y, que generen la transferencia de un conocimiento con significado para el participante y la sociedad.

De allí que la finalidad más importante en la enseñanza de la educación ambiental es lograr el desarrollo de sus potencialidades, así como el entendimiento y sensibilización ciudadana a través de la formación y participación de los seres humanos en actividades para el mejoramiento de la comunidad de tal manera que se interesen por mejorar el ambiente, resguardarlo del deterioro de sus recursos naturales y que a su vez obtengan conocimientos, aptitudes y actitudes para mantenerlo en condiciones óptimas para las próximas generaciones.

Se considera ciertamente que, la mayor dificultad en el desarrollo de los contenidos programáticos en el proceso de enseñanza y aprendizaje de la educación ambiental, posiblemente se encuentre en el modo de llevar la integración didáctica del conocimiento a la práctica y acción. Comprender realmente qué es, internalizar que no es un campo de estudio, sino un proceso, que para algunos se hace difícil entenderlo. Estas razones obligan a la revisión y a la incorporación de acciones que permitan el abordaje e integración didáctica de la enseñanza de la educación ambiental contribuyendo a una vinculación práctica en la formación que impacte hacía una actuación del estudiante, del profesor y de la ciudadanía en general hacia la sociedad.

Razón por la cual surge la motivación de la investigadora para realizar un programa referente a la integración didáctica de los contenidos ambientales desarrollados en el primer grado del Grupo Escolar María La Rivas Salas, 
ubicado en el Municipio Barinas Estado Barinas, a los fines de proponer una alternativa efectiva para solventar la problemática descrita, razón por la cual con la presente investigación se propone dar respuesta a los siguientes interrogantes:

¿Qué conocimiento tienen los docentes para la integración didáctica de los contenidos ambientales desarrollados en el primer grado del Grupo Escolar María La Rivas Salas, ubicado en el Municipio Barinas Estado Barinas?

¿Cuáles significados y sentidos emergentes ofrece la educación ambiental desde su didáctica y práctica de enseñanza?

¿Se considera necesario el diseño de un programa para la integración didáctica de los contenidos ambientales desarrollados en el primer grado del Grupo Escolar María La Rivas Salas?

A partir de estas preguntas se estipulan los objetivos de la investigación.

Para dar respuestas a dichas interrogantes se formula el Objetivo General de la Investigación donde el mismo se estableció como alternativa para la solución de la problemática ambiental relacionada a la establecer un programa para la integración didáctica de los contenidos de la educación ambiental, en el primer grado del plantel, cuya ejecución contribuye a mejorar la calidad de vida de los y las educandos.

Es importante resaltar que el Programa para la Integración Didáctica de los Contenidos de Educación Ambiental responde a la línea de investigación: Educación, Desarrollo y Gestión Comunitaria de la Universidad Pedagógica Experimental Libertador (UPEL).

\section{Teoría y Conceptos}

Los aspectos considerados en este capítulo, de acuerdo a lo planteado por Arias (2006), corresponde "al producto de la revisión bibliográfica y documental realizada por el investigador con el fin de precisar y construir, con 
base en diferentes posturas y definiciones, un sustento sólido para el desarrollo de la investigación" (pág. 26).

Esto implica que la documentación tiene relación con investigaciones y aportes teóricos de diferentes autores, que contribuyen en la consolidación y argumentación de los constructos bases del programa, además se abordan los preceptos filosóficos y legales significativos para el estudio.

\subsection{Antecedentes Investigativos}

Para la presente investigación se tomaron como base los siguientes estudios que se vinculan con el contenido del tema, sirviendo como referentes, a saber: Al respecto, Montes (2012), en su trabajo titulado "Aspectos teórico metodológicos en la didáctica de enseñanza de la educación ambiental en la Institución Educativa Antonio José de Sucre, ubicada en Bogotá, D. C.", el cual tuvo como objetivo analizar los aspectos teórico metodológicos en la didáctica de enseñanza de la educación ambiental en la Institución Educativa Antonio José de Sucre, ubicada en Bogotá, D. C.

Como conclusiones el autor expuso que, no existe vinculación entre la dimensión teórica y práctica en la enseñanza de la educación ambiental en la institución objeto de estudio; por lo cual la didáctica es de tipo determinista y tradicional no desarrollando un aprendizaje significativo en los educandos. Por otra parte, los docentes carecen de herramientas y estrategias didácticas para el abordaje de la temática ambiental, que logre vincularla con la realidad social $\mathrm{y}$ de vida de las personas.

Así mismo Cañizales (2011), en su trabajo titulado "Didáctica para la enseñanza de la educación ambiental como eje articulador en el seminario de desarrollo endógeno para el colectivo educativo de la etapa diversificada del Liceo Bolivariano José Rafael Pulido Méndez del Municipio Pedraza del Estado Barinas", la cual tuvo como objetivo analizar la didáctica para la enseñanza de la educación ambiental como eje articulador en el seminario de 
desarrollo endógeno para el colectivo educativo de la etapa diversificada del Liceo Bolivariano José Rafael Pulido Méndez del Municipio Pedraza del Estado Barinas, para el año 2011.

Como conclusiones se obtuvo que, una vez tabulado y analizados los resultados se evidenció la ausencia en el desarrollo de proyectos, actividades y estrategias que fomente en los estudiantes la enseñanza de la educación ambiental, la conservación del ambiente y el desarrollo endógeno, se observó el alto interés del estudiantado por involucrarse en estas actividades, lo cual justifica la recomendación de diseño de acciones desde la educación ambiental como eje articulador en el seminario de desarrollo endógeno.

Estos estudios planteados sirvieron de base de la investigación, debido a que aportaron elementos teóricos que sustentaron la didáctica en la enseñanza de la educación ambiental y a su vez para ampliar la disertación en cuanto a la integración didáctica de contenidos en el proceso de enseñanza y aprendizaje de la educación ambiental.

De igual manera estos estudios guardas relación con la presente investigación en función de considerar la necesidad de articulación de los contenidos que se desarrollan en la implementación de la educación ambiental desde un punto de vista didáctico y práctico, por lo que se orienta a la participación y el protagonismo de los sujetos de la comunidad educativa bajo los principios de corresponsabilidad, en la que intervienen todos los actores del hecho educativo; estas afirmaciones sustentan la argumentación investigativa y las bases teóricas del estudio.

\subsection{Referentes Teóricos}

En este sentido, Martínez (2007), plantea que éstas "representa el conjunto de definiciones y aportes documentales que permitan darle consistencia a cada uno de los enfoques para explicar la situación referida 
dentro del marco contextual del estudio" (pág. 45), es decir, las bases teóricas permiten el desarrollo de las variables contenidas en la investigación.

\subsubsection{Programa}

El programa constituye uno de los elementos claves para que pueda darse, satisfactoriamente, la participación e integración de las actividades en un contexto determinado, mientras que la planificación es una herramienta que permite la formulación y puesta en marcha de estrategias en función de la misión de los colectivos, objetivos, del medio ambiente sus presiones y de los recursos disponibles.

Al respecto Soler (2010), define que un plan representa "el proceso por el cual los dirigentes ordenan sus objetivos y acciones en el tiempo. No es un dominio sino un proceso de comunicación y de determinación de decisiones en el cual intervienen todos los niveles de la organización" (pág. 44). De acuerdo a lo antes expuesto, tanto el programa como la planificación se presentan como una guía de trabajo, abierto y flexible que forma parte de la función educativa social que integra todas las acciones realizadas en la institución, garantizando el desarrollo de medidas habilitadoras y compensadoras dirigidas a un contexto, concretándose en planes de actuación.

\subsubsection{Integración Didáctica de los Contenidos de la Educación Ambiental}

La educación ambiental ha surgido como tal y ha tomado auge en las últimas décadas, representa un campo en construcción que se puede desarrollar de manera individual y colectiva. La práctica educativa ambiental se sitúa en una perspectiva más amplia, que permite concretar los objetivos a desarrollar, no olvidando ninguno de los ámbitos: cognitivo, afectivo, holístico, de relación interpersonal y de actuación e inserción social, acercando al educando a la verdadera dimensión de los problemas ambientales. 
Refiere Álvarez (2012), "la introducción de la temática ambiental en el sistema educativo exige un nuevo modelo de profesor" (pág. 90). Por tanto, la capacitación del docente es indispensable para lograr el cambio que se propone la enseñanza de la educación ambiental y permitir su desempeño como educador transformador de su propia práctica, las de sus educandos y de la comunidad.

\subsubsection{Conceptualización de la Educación Ambiental}

La educación ambiental según Nieves (ob.cit.), se sitúa en "un proceso que conduce alcanzar una visión compleja y comprometida de la realidad, educar para una nueva forma operativa entre la realidad y el medio ambiente" (pág. 32). Esto supone la incorporación de cambios conceptuales y metodológicos, implica el desarrollo de habilidades, actitudes y valores que expresan ideas, emiten opiniones razonadas, promueven el trabajo cooperativo y la toma efectiva de decisiones.

El Ministerio del Poder Popular para el Ambiente (2009), define la educación ambiental en Venezuela como "el proceso que posibilita la formación de un hombre capaz de comprender la complejidad producida en el ambiente por la interacción de sus componentes naturales y socioculturales" (pág. 16).

En este sentido, el autor antes referido, expresa la capacidad crítica del individuo a los fines de emitir juicios de valor y adoptar normas de comportamiento para mejorar en forma constante las condiciones de vida. En el contexto de la educación bolivariana se tiene como objetivo sensibilizar, capacitar y formar en educación ambiental al docente bolivariano y otros participantes, a través de un programa continuo de acciones educativas: talleres, cursos y encuentros, entre otros, para la protección de la diversidad biológica que garanticen el desarrollo sustentable (Currículo Básico Nacional: (2007). 
Así mismo, la educación ambiental en el marco educativo venezolano, tiene como finalidad, proporcionar a docentes las bases conceptuales y metodológicas que les permitan promover proyectos educativos comunitarios. Desde esta perspectiva, resulta evidente, que la educación ambiental es concebida como política educativa, que orienta el diseño curricular para la educación básica de Venezuela, al promover contenidos ambientales que muestren las causas y las consecuencias de las condiciones de vida de la población.

\section{Metodología}

En esta etapa se presenta la descripción de la metodología a utilizar en la investigación, es decir, el conjunto de métodos y técnicas para explicar el presente estudio de investigación.

\subsection{Naturaleza de la Investigación}

Desde el punto de vista epistemológico y metodológico, la investigación corresponde en su naturaleza al enfoque cualitativo. Debido a que se trata de comprender a las personas dentro del marco de referencia de ellas mismas, de tal manera que es especial experimentar la realidad de como otros la experimentan.

\subsection{Tipo de investigación}

De acuerdo al tipo de investigación corresponde a la Investigación Acción Participación, que viene adaptando las pautas establecidas por el modelo metodológico propuesto por Castillo (2005), quien afirma que, "la investigación acción participación, tiene su raíz en el paradigma cualitativo y el mismo constituye un proceso continuo, e integrado donde se van dando las fases que se integran, para luego reiniciar un nuevo circuito" (pág. 32). A partir 
de las ideas propuestas por este autor, el esquema estuvo constituido por las siguientes fases:

1.- Problematización: la investigación se inicia a partir de una situación práctica, donde lo básico no es sólo conocerla, sino profundizarla en su significado y reflexionarla, de porqué era una necesidad considerada en el colectivo de abordar, cuáles son sus términos, características, y el contexto en el cual se produce.

La identificación de todos estos aspectos posibilita una contextualización clara del objeto de estudio, propiciando la motivación de los involucrados hacia el cambio que se desea obtener.

2.- Diagnóstico: una vez identificado el significado y contextualización del objeto de estudio, formulado el enunciado del mismo, se realiza la recolección de la información necesaria para obtener un diagnóstico preciso de la situación.

Esta recopilación debe recoger hechos, evidencias, expresiones y opiniones, desde los diferentes puntos de vista, de las personas implicadas, más que un análisis o deconstrucción lineal del todo en sus partes, se procura una interpretación o construcción de construcción del todo en sus partes y de las partes en un todo, es decir, se permite detentar una actitud hermenéutica ante lo observado.

3.- Diseño de una Propuesta de Cambio: luego de analizada e interpretada la información, se procede a pensar y visualizar diversas alternativas de actuación, en donde éstas permitieran diseñar y aplicar una propuesta de cambio, cuyas actividades, bien articuladas, le darán forma y estructura a la investigación.

Esta propuesta considera objetivos a lograr, recursos necesarios, lapsos en los cuales se ejecutaría, responsable de cada acción; igualmente, define su evaluación, es decir, los indicadores que darán cuenta de su logro. 


\subsection{Actores Sociales}

Los actores sociales son todas aquellas personas, que cuentan con un conocimiento acerca del medio o problema sobre el que se va a realizar el estudio. Para abordar la investigación se considera como unidad de estudio a los tres (03) docentes del primer grado del Grupo Escolar María La Rivas Salas del Municipio Barinas Estado Barinas.

Al respecto, es definida por Hernández (2007), "como la totalidad del fenómeno a estudiar en donde las unidades de población (personas o cosas) poseen una característica común, la cual se estudia y dan origen a los datos de la investigación" (pág. 60). Es decir, que los docentes de allí constituyen el grupo que manifestó la realidad que se vive.

\subsection{Técnica e instrumentos de recolección de datos}

Los procedimientos metodológicos deben orientarse según la posición de Martínez (ob.cit.), "hacia el descubrimiento de las estructuras personales o grupales, siendo necesario tener presente las formas que se revelan 0 expresan dichas estructuras" (pág. 62). Las técnicas más usadas para recabar la información se centran comúnmente en el lenguaje hablado o escrito. En el caso del presente estudio que se ocupa, se utiliza la técnica de la observación participante a través de notas de campo y la entrevista en profundidad de tipo no estructurada.

La observación participante, de acuerdo a la postura de Claret (2009), se define como la técnica" sustentada en el análisis y registro del comportamiento de la persona, objeto, unidad o acontecimiento a investigar" (pág. 177). Es decir, el investigador debe vivir lo más que pueda con las personas o grupos que desea investigar compartiendo sus usos, costumbres, estilos y modalidades de vida.

Al hacer referencia a la entrevista en profundidad, como técnica de recolección de información, se destaca, según Gómez (2006), que es "el 
encuentro cara a cara entre el investigador y los informantes claves" (pág. 62). Es decir, la que adopta la forma de un dialogo que describe en profundidad los procesos o estructuras que no se observan a simple vista, si no que se relatan.

\section{Análisis De Los Resultados}

En consecuencia, se presentan los resultados correspondientes, tomando como referencia la categorización, análisis e interpretación de datos y a que la información fue organizada mediante la clasificación de los contenidos en categorías y subcategorías; por su parte la triangulación de datos consiste en comparar y contrastar la información derivada de la observación y la entrevista en profundidad, provenientes de diferentes fuentes de información suministrada por los informantes claves.

De allí que, a partir de la categorización y triangulación se establecieron vínculos y relaciones entre los diferentes hechos registrados en el contexto estudiado. En cuanto al diseño de la propuesta relacionada a Acciones Estratégicas para el Fortalecimiento de los Patios Productivos como alternativa del Desarrollo Sustentable, contempló las siguientes Etapas: I Sensibilización, II Diagnóstico, III Capacitación y IV Evaluación, Control y Monitoreo con acciones que serán operacionalizadas de acuerdo a las necesidades, recursos humanos, estrategias y acciones en función a la temática objeto de estudio.

Cabe resaltar que el mismo permitió presentar a los docentes un conjunto de estrategias que permiten la integración didáctica de la educación ambiental con otras áreas de conocimiento, fortaleciendo en el proceso de enseñanza y aprendizaje, que a la vez los hace vincularlos y hacer partícipe a los padres y representantes en el logro de conocimientos y desarrollo de habilidades.

Es importante destacar que todos los objetivos previstos fueron logrados, dando cumplimiento con todas las actividades planificadas, algunas

de ellas fueron alteradas en relación a la fecha que se habían pautado, 
motivado a imprevistos ante la realización de dichos eventos. Durante la ejecución de las actividades se pudo lograr la participación y la organización de la comunidad educativa para el desarrollo de las actividades.

\subsection{Conclusiones}

Una vez desarrollada y ejecutada la investigación, se presentan las conclusiones que dieron a lugar en función de los resultados y de los objetivos planteados, de tal manera se presentan: En cuanto al diagnóstico desconocimiento que tienen los docentes para la integración didáctica de los contenidos ambientales desarrollos en el primer grado del Grupo Escolar María La Rivas Salas, ubicado en el Municipio Barinas Estado Barinas, se obtuvo que: El proceso de evaluación fue cualitativo, a partir de las expresiones realizadas por los docentes participantes quienes manifestaron la efectividad de los talleres, considerándolos satisfactorios $y$, recordaron que esta labor debe continuar. En relación a la interpretación de los significados y sentidos emergentes que ofrece la educación ambiental desde su didáctica y práctica de enseñanza, se obtuvo que: La triangulación de las opiniones emergentes en cuanto a la aplicación de estrategias didácticas. Referente al impacto educativo, las opiniones emergentes sostienen que las estrategias didácticas, así como la actuación del docente no son motivadoras para el proceso de enseñanza y aprendizaje e integración de la educación ambiental. Es importante resaltar que para finalizar los resultados obtenidos se evidenciaron debilidades en cuanto a las estrategias del docente en la planificación de aprendizajes basados en el nivel de educación primaria para integrar los contenidos ambientales con otras áreas del conocimiento, por lo tanto, la presente propuesta reviste importancia para incorporar acciones estratégicas que abarcan desde el carácter tecnológico hasta el didáctico participativo. Es de hacer notar que las acciones presentadas, proporcionan el inicio de un sinfín de otras que el docente puede practicar de forma significativa 
y durante el año escolar, ya que, la continuidad en la ejecución de las mismas producirá cambios positivos en los aprendizajes.

\subsection{Recomendaciones}

Es importante resaltar que la presente investigación guarda relación con el siguiente artículo de la Autora: González Castillo, M. (2017). Estrategias Didácticas para el Fortalecimiento de los Valores Ambientalistas. Revista Scientific, 336-354. Consultado en línea de:

http://www.indteca.com/ojs/index.php/Revista_Scientific/article/view/81

Posterior a la presentación de las conclusiones, se procede a formular algunas recomendaciones destinadas para contribuir a desarrollar estrategias en la planificación de aprendizajes del nivel del subsistema de educación primaria:

1.- Considerar la aplicación de esta propuesta dentro de la planificación educativa de la institución.

2.- Conformar espacios de intercambio entre el personal docente sobre estrategias didácticas y tecnológicas, que resultaron deficientes, a través de círculos de estudios, que permitan intercambiar las experiencias y fortalecer las habilidades para mejorar las estrategias a utilizar en el proceso de enseñanza y aprendizaje respondiendo a las necesidades de impulsar el crecimiento del individuo $y$, del colectivo a través de aprendizajes significativos.

3.- Estimular la práctica continua mediante las formas de organización como el Proyecto Educativo Institucional y el Proyecto de Desarrollo Endógeno, para desarrollar estrategias que permitan mejorar la planificación y la participación de manera integrada de todos los actores en el proceso educativo. 
4.- Promover en el docente la formación y capacitación sobre actuaciones tecnológicas y didácticas.

5.- Ofrecer al personal docente capacitación sobre estrategias tecnológicas para ser utilizadas por el docente en la planificación de aprendizajes.

6.- Desarrollar actividades que le permitan al docente elaborar actuaciones didácticas acorde a las características de sus educandos para mejorar el aprendizaje en la educación primaria.

\section{Referencias}

Álvarez, P. (2012). Propuesta Didáctica para la Elaboración de Manual sobre la Educación Ambientalista en la Enseñanza del Subsistema de Educación Primaria Venezolano. Caso Estudio: Unidad Educativa La Coromoto, Municipio Mario Briceño Irragoren en el Estado Aragua. Trabajo de Grado. Universidad Bicentenaria de Aragua.

Arias, F. (2006). El proyecto de investigación: Introducción a la Metodología Científica. (5ta. ed.) Caracas. Venezuela: Episteme.

Claret, V. (2009). Proyectos Comunitarios e Investigación Cualitativa. (6ta ed.). Caracas, Venezuela.

Cañizales, K. (2011). Didáctica para la enseñanza de la educación ambiental como eje articulador en el seminario de desarrollo endógeno para el colectivo educativo de la etapa diversificada del Liceo Bolivariano José Rafael Pulido Méndez del Municipio Pedraza del Estado Barinas. Trabajo de Grado. UNELLEZ.

Castillo, A. J. (2005). Formación del profesorado en Educación Superior. Madrid, España McGraw-Hill

Gómez, U. (2.006). Pertinencia de la investigación que se realiza en los Institutos Universitarios de Tecnología de la Región Centro Occidental de Venezuela. 
Hernández, R. (2007). Metodología de la Investigación. México: McGrawHill.

Ministerio del Ambiente y de los Recursos Naturales (2009). Caracas. Venezuela.

Ministerio del Poder Popular para la Educación (2007). Currículo Básico Nacional Bolivariana. Caracas, Venezuela.

Manual de Trabajo de Grado de Especialización, Maestría y Tesis Doctorales. (1998). FUNDAUPEL. Caracas, Venezuela.

Martínez, H. (2007). La Investigación cualitativa. España. Reedic.

Montes, P. (2012). Aspectos teórico-metodológicos en la didáctica de enseñanza de la educación ambiental en la Institución Educativa Antonio José de Sucre, ubicada en Bogotá, D.C. Trabajo de Investigación. Universidad de Santander.

Nieves, P. (2011). El desafío ambiental. Ecología y medio ambiente. Transversales Magisterio. Bogotá.

Pérez, A. (2009). Método cualitativo. Colección Ensayos, San Pablo. Venezuela San Pablo.

Salas, M. (2009). La educación ambiental en la educación básica, desde el paradigma de la complejidad. Trabajo de Tesis Doctoral publicado. Universidad del Zulia.

Sandoval, P. (2012). Cómo construir un programa de educación ambiental. Madrid: Los Libros de la Catarata. 


\section{Johana Carolina González González \\ e-mail: johanavdsg@hotmail.com}

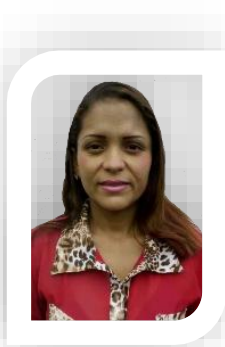

Nacida en Barinas, Estado Barinas, Venezuela. Licenciada en Educación Integral, graduada en la Universidad Nacional Experimental de los Llanos Occidentales Ezequiel Zamora (UNELLEZ), Barinas. (2006). Soltera. Docente III. Estudiante de la Maestría en Educación Ambiente y Desarrollo en la Universidad Pedagógica Experimental Libertador (UPEL), Barinas. Trabajo en el Ministerio del Poder Popular para la Educación (MPPPE). Pertenezco a la nómina y laboro actualmente en el Grupo Escolar "María la Riva Salas", como Docente de Aula en el Subsistema de Educación Primaria, en Barinas Estado Barinas.

El contenido de este manuscrito se difunde bajo una Licencia de Creative Commons ReconocimientoNoComercial-Compartirlgual 4.0 Internacional 